\title{
Rapid identification of Candida glabrata in Candida bloodstream infections
}

\author{
Correspondence \\ Nicholas Foster \\ NicholasFoster@Doctors.org.uk
}

Received 21 May 2007

Accepted 15 August 2007

\author{
Nicholas Foster, Charlotte Symes, Richard Barton and Richard Hobson \\ Department of Mycology, The Old Medical School, Thorseby Place, Leeds LS2 9JT, UK
}

\begin{abstract}
Candida species are the fourth most common cause of bloodstream infection (BSI) in the hospitalized patient. Candida glabrata is the most common non-Candida albicans Candida species in England and Wales with an attributed mortality of $48 \%$. C. glabrata is known to demonstrate reduced susceptibility to fluconazole, resulting in treatment failures when employing this agent for empirical treatment of Candida BSI. The first part of this study demonstrated a technique utilizing a blood culture system commonly used by many laboratories (BACTEC 9240 automated detection system) that reduced the time to identification of this potentially resistant organism by up to $72 \mathrm{~h}$. A presumptive identification was achieved by observing a difference in the duration of incubation required before growth was detected automatically between Lytic Anaerobic and Plus Aerobic culture bottles. Secondly, experiments exploring the growth characteristics of C. glabrata in BACTEC blood culture bottles containing various media were carried out to explore possible reasons underpinning this clinical observation. The detection of yeast in the anaerobic bottle of a blood culture pair consisting of Lytic Anaerobic and Plus Aerobic in a BACTEC 9240 system was found to be highly predictive of the isolation of C. glabrata (positive predictive value $93.3 \%$, negative predictive value $98.3 \%$ ). The reason for this appeared to be a component of the Lytic Anaerobic blood culture medium enhancing the growth of $C$. glabrata in that medium.
\end{abstract}

\section{INTRODUCTION}

Candida species are the fourth most common cause of bloodstream infection (BSI) in the hospitalized patient (Edmond et al., 1999; Hobson, 2003) with an attributed mortality of $38 \%$ (Wey et al., 1988). The risk factors predisposing to this condition are host-related (such as immunosuppression) and healthcare-related (such as presence of intravascular catheters, use of broad-spectrum antibiotics, gastrointestinal surgery, total parenteral nutrition and intensive care admission) (Clark \& Hajjeh, 2002; Hope et al., 2002). Although the overall incidence of Candida BSI is reported to be falling (Hobson, 2003; Kibbler et al., 2003), the proportion of BSIs caused by nonCandida albicans Candida species, in particular Candida glabrata, is increasing (Hope et al., 2002; Maschmeyer, 2006; Trick et al., 2002). BSI with this species increases in incidence with patient age (Diekema et al., 2002) and has an associated mortality of $48 \%$ (Maschmeyer, 2006).

In England and Wales, C. albicans remains the most common Candida bloodstream isolate, accounting for $64.7 \%$ of cases. C. glabrata is the second most frequently isolated Candida species from blood, comprising $16.2 \%$ of

Abbreviations: BSI, bloodstream infection; CPDA, citrate/phosphate/ dextrose/adenine; TTP, time to positivity. all Candida species (Kibbler et al., 2003). C. glabrata has been found to have a reduced susceptibility to fluconazole (60\% sensitive, $31 \%$ dose-dependent sensitive, 9\% resistant) (Pfaller et al., 2003), resulting in treatment failure when using fluconazole for the empirical management of haematogenous or deep-seated Candida infections (Lee et al., 2000; Rex et al., 1997). This is important as, when identifying Candida species using current gold standard techniques (API ID 32C, bioMérieux; or Auxacolor 2, Bio$\mathrm{Rad})$, a delay of $48-72 \mathrm{~h}$ can be expected before a result is available (according to the product instructions of the manufacturers). The current Infectious Diseases Society of America Guidelines for Treatment of Candidaemia highlight the importance of rapid speciation in order to predict antifungal susceptibility (Pappas et al., 2004). Due to the reduced susceptibility of C. glabrata to fluconazole, caspofungin is suggested as a good alternative when an infection with this species is suspected (Pappas et al., 2004). This paper describes a technique that could presumptively identify C. glabrata as the causative organism as soon as a yeast BSI is diagnosed. This would be a great benefit to treatment planning in the interim, before antimicrobial sensitivities are made available and without the delay resulting from the traditional identification procedures.

It was noted in our institution that isolates of C. glabrata from patient specimens were being detected initially from 
the anaerobic bottle of the blood culture set. Horvath et al. (2003), in a simulated candidaemia experiment, also demonstrated a significantly shorter incubation time to detection of C. glabrata in the anaerobic bottle from blood culture sets inoculated with Candida species and incubated in the Becton Dickinson BACTEC 9240 automated detection system. This phenomenon was also noted by Fitzgibbons et al. (2001) in a retrospective analysis of blood culture specimens submitted to the laboratory for investigation and reported in abstract form. However, a report of the utility of this finding in the clinical setting has not been published.

In the first part of this study, clinical laboratory data were analysed retrospectively and demonstrated that a presumptive identification of $C$. glabrata could be made immediately on diagnosis of a yeast BSI. A presumptive identification was achieved by observing a difference in the duration of incubation required before growth was detected automatically between the Lytic Anaerobic and Plus Aerobic culture bottles.

Secondly, the growth characteristics of C. glabrata in BACTEC blood culture bottles containing various media were compared to explore possible reasons for the clinical laboratory observation.

\section{METHODS}

Retrospective data analysis. Blood cultures were taken using aseptic technique from patients whose clinical features were suggestive of BSI. Blood was inoculated into BACTEC Lytic Anaerobic and Plus Aerobic bottles (Becton Dickinson), transported to the laboratory and loaded onto the BACTEC 9240 automated detection blood culture system.

The BACTEC 9240 automated detection blood culture system detected growth in the bottles and alerted the laboratory staff by means of an audible alarm. The time taken from loading each bottle on to the machine to the detection of growth (time to positivity, TTP) was recorded for each bottle in the set. Medium $(0.5 \mathrm{ml})$ was removed from each positive blood culture bottle for Gram staining. Positive blood culture bottles from which yeast cells were observed following Gram staining were subcultured onto Columbia chocolate agar (E \& O Laboratories) (incubated at $37{ }^{\circ} \mathrm{C}$ in $5-10 \% \mathrm{CO}_{2}$ ) and Sabouraud's dextrose agar (E \& O Laboratories) (incubated at $37{ }^{\circ} \mathrm{C}$ in air) and monitored daily for growth. Cultures of yeast species on either medium were referred to the Mycology Reference Centre (Leeds, UK) for identification. All isolates were subcultured on CHROMagar Candida medium (M-Tech Diagnostics) and a germ tube test was performed (incubation of yeast cells in $0.5 \mathrm{ml}$ calf serum at $37{ }^{\circ} \mathrm{C}$ for $1.75 \mathrm{~h}$ ). Yeasts producing germ tubes were identified as C. albicans unless they were observed to have dark-green colonies on CHROMagar Candida medium. Dark-green colonies were tested for filamentation on Niger seed agar (produced in-house) to determine whether the isolate was Candida dubliniensis (Lees \& Barton, 2003). Candida species not producing germ tubes were identified using Auxacolor 2 colorimetric sugar assimilation tests (Bio-Rad Laboratories) and microscopic morphology following subculture on Dalmau agar (produced in-house). Candida species that could not be identified with this technique were identified using the API ID 32C identification system (bioMérieux). Blood culture bottles in which no growth was detected were incubated for a total of 5 days before being discarded as negative.
A search was performed using the Telepath Laboratory Management System (iSoft). Specimens from the Leeds Teaching Hospitals Trust and Bradford Hospitals NHS Trust between July 2004 and June 2006 were included.

Positive investigations were selected from all blood cultures sent to the laboratory for routine clinical investigation of suspected BSI. A 'positive investigation' was defined as a blood culture with growth detected and a Gram film or culture growth with yeast or suspected yeast. Specimens were excluded if: (i) an incomplete set was submitted (Lytic Anaerobic or Plus Aerobic only); (ii) a single paediatric bottle was submitted; or (iii) the Gram film appearance was mixed or did not demonstrate a yeast.

The hypothesis under test was that the organism isolated from a blood culture set consisting of Lytic Anaerobic and Plus Aerobic bottles would be C. glabrata if (i) the first bottle in which growth was detected was the anaerobic bottle and (ii) the Gram film identified yeasts only. Blood culture results were categorized as 'test positive' where these criteria were met and 'test negative' where they were not. The blood cultures were then analysed according to the yeast species isolated (C. glabrata or other Candida species). Specimens that grew a mixture of organisms on subculture were included in the 'Glabrata' group if C. glabrata was isolated from the mixture.

Prospective laboratory investigation. Four randomly selected strains of C. glabrata from patient specimens from the above group were subcultured on Sabouraud's dextrose agar and incubated at $37{ }^{\circ} \mathrm{C}$ in room air. After $24 \mathrm{~h}$ incubation, a single colony of each was inoculated into $10 \mathrm{ml}$ Sabouraud's liquid medium (reconstituted in sterile distilled water and autoclaved on site; Oxoid) and incubated for $48 \mathrm{~h}$ on a shaking tray at $37^{\circ} \mathrm{C}$ in room air to attain a steady-state culture. A cell count was performed using a Füchs-Rosenthal counting chamber and each broth culture was diluted in sterile saline $(0.9 \%, \mathrm{w} / \mathrm{v})$ to obtain concentrations of $10^{2}$ and $10^{3} \mathrm{cells}^{-1}$. The dilutions were confirmed by viable count: Sabouraud's dextrose agar was inoculated with $100 \mu \mathrm{l}$ of the dilutions, incubated for $24 \mathrm{~h}$ at $37{ }^{\circ} \mathrm{C}$ in room air and the colony numbers were recorded.

A range of BACTEC blood culture bottles were used to elucidate the growth preferences of C. glabrata. Lytic Anaerobic, Plus Aerobic, Plus Anaerobic, and both Lytic Anaerobic and Plus Aerobic with the bottle atmosphere replaced with room air (vented) were used to investigate the role of both medium and atmosphere composition. Each of the five blood culture bottles was inoculated aseptically with $10 \mathrm{ml}$ waste whole blood (National Blood Service, Bridle Path, Leeds, UK), which had been collected from healthy volunteer donors into transfusion bags pre-filled with CPDA (citrate/phosphate/dextrose/adenine) anticoagulant (Baxter Healthcare). C. glabrata suspension (1 ml) was introduced into each bottle. There were four replicates for each

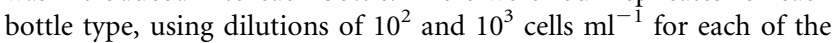
four C. glabrata strains (a total of 40 results for each bottle type). Following inoculation, the bottles were incubated using the BACTEC 9240 automated detection blood culture system. The TTP was recorded for each sample.

After flagging positive, $10 \mu \mathrm{l}$ medium was removed, subcultured on Columbia chocolate agar and incubated for $48 \mathrm{~h}$ at $37^{\circ} \mathrm{C}$. Cultures were checked for pure growth of Candida species. Cultures with bacterial or mixed growth were excluded from the results.

Statistical analysis. For the retrospective data analysis, Microsoft Excel software was used to calculate the sensitivity, specificity, positive predictive value and negative predictive value of the test.

The data for the laboratory investigation were analysed with SPSs for Windows (version 9.0; SPSS). A one-way analysis of variance (ANOVA) for TTP of each bottle type was performed for the two inoculum densities $\left(10^{2}\right.$ and $10^{3}$ cells $\left.\mathrm{ml}^{-1}\right)$. A Games-Howell 
post-hoc analysis calculated the significance of the differences between the mean TTP of each bottle. One-way ANOVA with Games-Howell post-hoc analyses of mean TTP for each of the four strain types was also performed to determine interstrain variability.

\section{RESULTS AND DISCUSSION}

\section{Retrospective data analysis}

A total of 328 positive investigations were identified. Of these, 87 investigations were excluded from analysis: 17 as a result of mixed Gram film appearance; 32 with no organisms seen in the Gram film, 24 with organisms other than yeasts seen in the Gram film, 11 containing fluid other than blood, and 3 with either no TTP recorded on the database or no identification of yeast to species level. Thus a total of 241 blood culture investigations were included in the analysis.

C. glabrata was isolated in 59/241 blood culture investigations (54 in pure culture and 5 mixed). The remaining yeast species isolated were C. albicans (97), Candida parapsilosis (44), Candida tropicalis (12), Cryptococcus neoformans (5), Candida guilliermondii (3), C. dubliniensis (2), Candida krusei (2), Candida lusitaniae (1) and Candida rugosa (1). Twenty blood cultures grew mixtures: $C$. albicans and bacteria (7); C. albicans and C. glabrata (3); C. glabrata and bacteria (2); C. parapsilosis and bacteria (3); C. tropicalis and bacteria (3); and Rhodotorula mucilaginosa and bacteria (2).

Initial detection of Candida spp. in the anaerobic bottle of a paired set of blood cultures (Lytic Anaerobic and Plus Aerobic) was found to be highly predictive of the subsequent isolation of C. glabrata, with a sensitivity of $94.9 \%$, specificity of $97.8 \%$, positive predictive value of $93.3 \%$ and negative predictive value of $98.3 \%$ (Table 1 ).

\section{Prospective laboratory investigation}

A single specimen (a vented Plus Aerobic bottle) from the experiment using an inoculum of $10^{3}$ cells $\mathrm{ml}^{-1}$ and four specimens (one Plus Anaerobic, one vented Plus Aerobic and two Plus Aerobic bottles) from the experiment using an inoculum of $10^{2}$ cells $\mathrm{ml}^{-1}$ were excluded from the analysis as a result of impurity of the terminal subculture.

Fig. 1 shows that the mean TTP was shortest in the Lytic Anaerobic (vented) bottles followed by Lytic Anaerobic,

Table 1. Results of the prospective laboratory investigation

\begin{tabular}{|lccr|}
\hline Identification & \multicolumn{3}{c|}{ Test hypothesis fulfilled } \\
\cline { 2 - 4 } & Positive & Negative & Total \\
\hline C. glabrata & 56 & 3 & 59 \\
Non-C. glabrata & 4 & 178 & 182 \\
Total & 60 & 181 & 241 \\
\hline
\end{tabular}

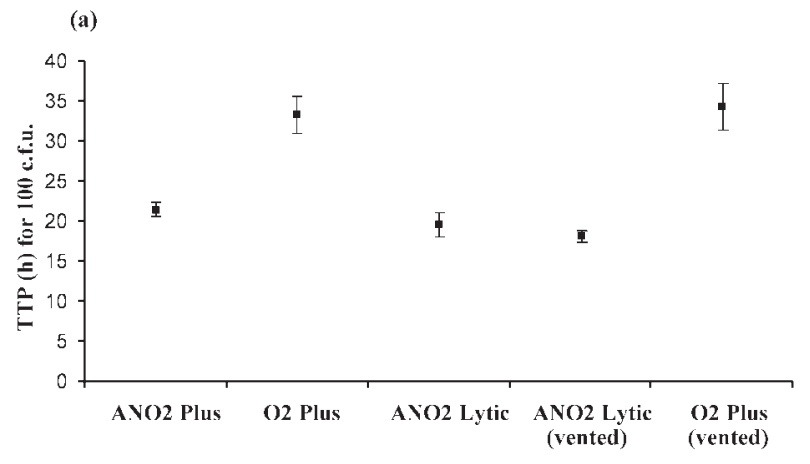

(b)

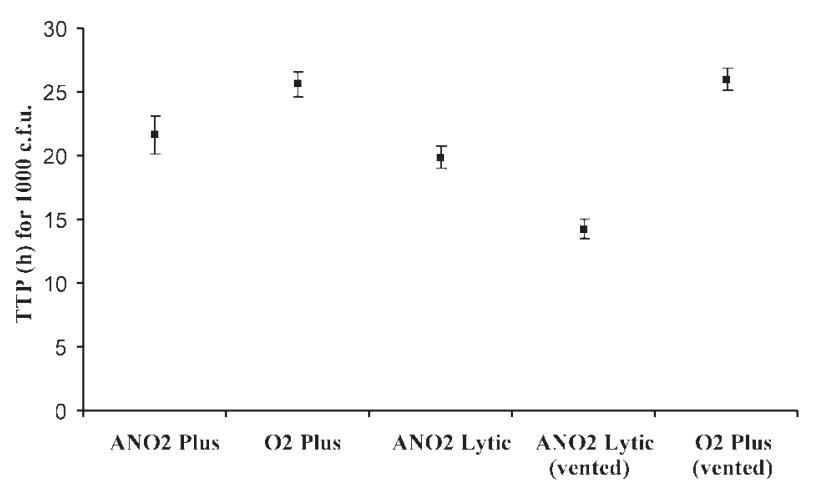

Fig. 1. Error plot demonstrating the mean TTP (h) ( \pm 2 SEM, for a $95 \%$ confidence interval) of the five bottle types inoculated with $10^{2}$ (a) or $10^{3}$ (b) cells $\mathrm{ml}^{-1}$. ANO2, anaerobic; O2, aerobic. Vented indicates that the bottle atmosphere could exchange with room air.

then the Plus Anaerobic followed by the Plus Aerobic, with the Plus Aerobic (vented) having the longest TTP for both inoculum concentrations.

The differences among bottle types with respect to mean TTP are shown in Table 2. For the experiment using an inoculum of $10^{2}$ cells $\mathrm{ml}^{-1}$, the Plus Anaerobic bottle showed a mean TTP significantly shorter than that of both the Plus Aerobic and Plus Aerobic (vented) $(P<0.001)$. There was no significant difference in TTP between the Plus Anaerobic and Lytic Anaerobic bottles $(P=0.241)$; however, the Lytic Anaerobic (vented) bottle was shown to have a significantly shorter TTP than the Plus Anaerobic $(P<0.001)$. Importantly, no significant difference was found between the Lytic Anaerobic and Lytic Anaerobic (vented) bottles $(P=0.442)$.

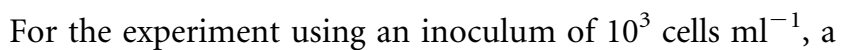
similar relationship was noted. However, a significant difference was identified between the Lytic Anaerobic and Lytic Anaerobic (vented) bottles $(P<0.001)$, but with a shorter TTP in the vented bottles. No significant difference was found between the Plus Aerobic and the Plus Aerobic (vented) bottles in either experiment. 
Table 2. Games-Howell post-hoc analysis comparing mean TTP among bottles

$P$ values in bold highlight statistically significant differences to a confidence value of $95 \%$.

\begin{tabular}{|c|c|c|c|c|c|}
\hline \multirow[t]{2}{*}{ Bottle (I) } & \multirow[t]{2}{*}{ Bottle $(J)$} & \multicolumn{2}{|c|}{$10^{2}$ cells $\mathrm{ml}^{-1}$} & \multicolumn{2}{|c|}{$10^{3}$ cells $\mathrm{ml}^{-1}$} \\
\hline & & $\begin{array}{l}\text { Mean difference } \\
(\mathrm{I}-\mathrm{J}) \text { in TTP }(\mathrm{h})\end{array}$ & $P$ value & $\begin{array}{l}\text { Mean difference } \\
(\mathrm{I}-\mathrm{J}) \text { in TTP }(\mathrm{h})\end{array}$ & $P$ value \\
\hline \multirow[t]{4}{*}{ ANO2 Plus } & O2 Plus & -11.7993 & $<0.001$ & -3.9720 & 0.001 \\
\hline & ANO2 Lytic & 1.8714 & 0.241 & 1.7640 & 0.261 \\
\hline & ANO2 Lytic (vented) & 3.3234 & $<0.001$ & 7.3680 & $<\mathbf{0 . 0 0 1}$ \\
\hline & O2 Plus (vented) & -12.7832 & $<0.001$ & -4.3579 & $<0.001$ \\
\hline \multirow[t]{3}{*}{ O2 Plus } & ANO2 Lytic & 13.6707 & $<0.001$ & 5.7360 & $<0.001$ \\
\hline & ANO2 Lytic (vented) & 15.1227 & $<0.001$ & 11.3400 & $<0.001$ \\
\hline & O2 Plus (vented) & -0.9839 & 0.983 & -0.3859 & 0.977 \\
\hline \multirow[t]{2}{*}{ ANO2 Lytic } & ANO2 Lytic (vented) & 1.4520 & 0.442 & 5.6040 & $<0.001$ \\
\hline & O2 Plus (vented) & -14.6545 & $<\mathbf{0 . 0 0 1}$ & -6.1219 & $<0.001$ \\
\hline ANO2 Lytic (vented) & O2 Plus (vented) & -16.1065 & $<\mathbf{0 . 0 0 1}$ & -11.7259 & $<0.001$ \\
\hline
\end{tabular}

The difference in mean TTP among the four strains for the experiment using an inoculum of $10^{3}$ cells $\mathrm{ml}^{-1}$ was not significant $(P=0.099)$ in any bottle. For the experiment using an inoculum of $10^{2}$ cells $\mathrm{ml}^{-1}$, there was a significant difference among strains $(P=0.039)$, with post-hoc analysis illustrating a difference between strain 1 and strain 2 $(P=0.03)$.

When utilizing the Becton Dickinson BACTEC 9240 automated system with the Lytic Anaerobic and Plus Aerobic culture bottle set, the isolation of yeast on Gram film initially from the anaerobic bottle was highly predictive $(93 \%)$ for the subsequent identification of $C$. glabrata. This observation reduced the time to identification by $48-72 \mathrm{~h}$ and is of benefit in early targeting of therapy towards this organism, $8 \%$ of isolates of which are resistant to fluconazole and a further $32 \%$ of which have reduced susceptibility to this agent (Pfaller et al., 2002). The retrospective data analysis confirmed the clinical observation, which will enable clinicians to initiate antifungal therapy appropriate for C. glabrata at an earlier stage of infection. It should be noted, however, that this observation was limited to a specific blood culture system (BACTEC 9240). Further work will be required if this observation is to be extrapolated to other systems. A prospective study looking at the impact of this observation on clinical outcome would be valuable in evaluating its significance in clinical practice.

The laboratory data investigating the TTP of the different blood culture bottles supported the conclusion that the phenomenon is caused by a constituent of the Lytic Anaerobic blood culture medium and is a chemical component of the blood culture medium, rather than atmospheric composition per se.

The Lytic Anaerobic (both vented and normal) and Plus Anaerobic bottles had a significantly shorter mean TTP than the Plus Aerobic (both vented and normal), with the exchange of atmosphere from anaerobic to room air in the
Lytic Anaerobic bottle further reducing the mean TTP. The exchange of atmosphere to room air in the Plus Aerobic

Table 3. Composition of the blood culture bottles used in this study

Bold denotes differing composition between aerobic and anaerobic bottles; ${ }^{\star}$ denotes unknown composition.

\begin{tabular}{|lccc|}
\hline Component & $\begin{array}{c}\text { Lytic } \\
\text { Anaerobic/F }\end{array}$ & $\begin{array}{c}\text { Plus } \\
\text { Aerobic/F }\end{array}$ & $\begin{array}{c}\text { Plus } \\
\text { Anaerobic/F }\end{array}$ \\
\hline Processed water (ml) & 40 & 25 & 25 \\
Soybean-casein digest & 2.75 & 2.75 & 2.75 \\
broth (\%) & & & \\
Yeast extract (\%) & 0.2 & 0.25 & 0.4 \\
Animal tissue digest (\%) & $\mathbf{0 . 0 5}$ & - & $\mathbf{0 . 0 5}$ \\
Dextrose (\%) & $\mathbf{0 . 2}$ & $\mathbf{0 . 0 6}$ & $\mathbf{0 . 2 5}$ \\
Sucrose (\%) & - & $\mathbf{0 . 0 8 4}$ & - \\
Fructose (\%) & - & - & 0.25 \\
Arginine (\%) & - & - & 0.25 \\
Haemin (\%) & 0.0005 & 0.0005 & 0.0005 \\
Menadione (\%) & 0.00005 & 0.00005 & 0.00005 \\
Pyridoxal HCl (\%) & - & $\mathbf{0 . 0 0 1}$ & - \\
Sodium citrate (\%) & $\mathbf{0 . 0 2}$ & - & $\mathbf{0 . 0 2}$ \\
Thiols (\%) & $\mathbf{0 . 1}$ & - & $\mathbf{0 . 1 6}$ \\
Sodium pyruvate (\%) & 0.1 & - & - \\
Potassium phosphate (\%) & - & - & 0.24 \\
Saponin (\%) & 0.26 & - & - \\
Antifoaming agent (\%) & $\mathbf{0 . 0 1}$ & - & $\mathbf{0 . 0 1}$ \\
Sodium & 0.035 & 0.05 & 0.05 \\
polyanetholsulfonate & & & \\
(\%) & & & \\
Nonionic absorbing resin & - & 16.0 & 16.0 \\
(\%) & & & \\
Cationic exchange resin & - & 1.0 & 1.0 \\
(\%) & & & \\
pH & $7.2 \pm 0.2$ & $7.2 \pm 0.2$ & $7.2 \pm 0.2$ \\
Atmosphere & $*$ & $*$ & $*$ \\
& & & \\
& & & \\
& & & \\
\hline
\end{tabular}


bottle had no impact on the mean TTP. This suggests that (i) C. glabrata has a predilection for a component of the Lytic Anaerobic and Plus Anaerobic media, and (ii) the provision of an aerobic atmosphere with this medium encourages further growth. Table 3 illustrates the differing medium components in the three bottles and offers a number of possibilities. It was noted that the simulated candidaemia experiments conducted by Horvath et al. (2003) resulted in a longer TTP for the Plus Aerobic bottles than the experiments in this study with the same inoculation concentrations, albeit with a differing method of inoculum preparation (mean TTP $25.57 \mathrm{~h}$ in this study vs $120.89 \mathrm{~h}$ in Horvath et al., 2003). A possible explanation may be found in the blood used to inoculate the media bottles. In the study conducted by Horvath et al. (2003), blood was collected from healthy volunteers and inoculated directly into bottles. In our study, availability of blood was restricted to samples collected from healthy volunteers and stored in bags pre-filled with CPDA anticoagulant. Two of these components can be found in the anaerobic but not the aerobic culture medium bottles. Further work is required to determine which, if any, of these components is responsible for these observations.

\section{REFERENCES}

Clark, T. A. \& Hajjeh, R. A. (2002). Recent trends in the epidemiology of invasive mycoses. Curr Opin Infect Dis 15, 569-574.

Diekema, D. J., Messer, S. A., Brueggemann, A. B., Coffman, S. L., Doern, G. V., Herwaldt, L. A. \& Pfaller, M. A. (2002). Epidemiology of candidemia: 3-year results from the emerging infections and the epidemiology of Iowa organisms study. J Clin Microbiol 40, 1298-1302.

Edmond, M. B., Wallace, S. E., McClish, D. K., Pfaller, M. A., Jones, R. N. \& Wenzel, R. P. (1999). Nosocomial bloodstream infections in United States hospitals: a three-year analysis. Clin Infect Dis 29, 239-244.

Fitzgibbons, N., Monahan, J. S., Fang, F. C. \& Madinger, N. E. (2001). Rapid presumptive identification of Candida glabrata using BACTEC Lytic/10 Anaerobic/F Medium. In Abstracts of the 41st Interscience Conference on Antimicrobial Agents and Chemotherapy, Chicago, Illinois, USA, 16-19 December. Abstract no. D-1393. Denver, CO: University of Colorado Health Science Center.

Hobson, R. P. (2003). The global epidemiology of invasive Candida infection - is the tide turning? J Hosp Infect 55, 159-168.

Hope, W., Morton, A. \& Eisen, D. P. (2002). Increase in prevalence of nosocomial non-Candida albicans candidaemia and the association of Candida krusei with fluconazole use. J Hosp Infect 50, 56-65.
Horvath, L. L., Hospenthal, D. R., Murray, C. K. \& Dooley, D. P. (2003). Detection of simulated candidemia by the BACTEC 9240 system with Plus Aerobic/F and Anaerobic/F blood culture bottles. J Clin Microbiol 41, 4714-4717.

Kibbler, C. C., Seaton, S., Barnes, R. A., Gransden, W. R., Holliman, R. E., Jonhson, E. M., Perry, J. D., Sullivan, D. J. \& Wilson, J. A. (2003). Management and outcome of bloodstream infections due to Candida species in England and Wales. J Hosp Infect 54, 18-24.

Lee, S. C., Fung, C. P., Huang, J. S., Tsai, C. J., Chen, K. S., Chen, H. Y., Lee, N., See, L. C. \& Shieh, W. B. (2000). Clinical correlates of antifungal macrodilution susceptibility test results for non-AIDS patients with severe Candida infections treated with fluconazole. Antimicrob Agents Chemother 44, 2715-2718.

Lees, E. \& Barton, R. C. (2003). The use of Niger seed agar to screen for Candida dubliniensis in a clinical microbiology laboratory. Diagn Microbiol Infect Dis 46, 13-17.

Maschmeyer, G. (2006). The changing epidemiology of invasive fungal infections: new threats. Int J Antimicrob Agents 27 (Suppl. 1), 3-6.

Pappas, P. G., Rex, J. H., Sobel, J. D., Filler, S. G., Dismukes, W. E., Walsh, T. J., Edwards, J. E. \& Infectious Diseases Society of America (2004). Guidelines for treatment of candidiasis. Clin Infect Dis 38, 161-189.

Pfaller, M. A., Messer, S. A., Hollis, R. J., Jones, R. N. \& Diekema, D. J. (2002). In vitro activities of ravuconazole and voriconazole compared with those of four approved systemic antifungal agents against 6,970 clinical isolates of Candida spp. Antimicrob Agents Chemother 46, 1723-1727.

Pfaller, M. A., Messer, S. A., Boyken, L., Tendolkar, S., Hollis, R. J. \& Diekema, D. J. (2003). Variation in susceptibility of bloodstream isolates of Candida glabrata to fluconazole according to patient age and geographic location. J Clin Microbiol 41, 2176-2179.

Rex, J. H., Pfaller, M. A., Galgiani, J. N., Bartlett, M. S., Espinel-Ingroff, A., Ghannoum, M. A., Lancaster, M., Odds, F. C., Rinaldi, M. G. \& other authors (1997). Development of interpretive breakpoints for antifungal susceptibility testing: conceptual framework and analysis of in vitro-in vivo correlation data for fluconazole, itraconazole, and Candida infections. Subcommittee on Antifungal Susceptibility Testing of the National Committee for Clinical Laboratory Standards. Clin Infect Dis 24, 235-247.

Trick, W. E., Fridkin, S. K., Edwards, J. R., Hajjeh, R. A., Gaynes, R. P. \& the National Nosocomial Infections Surveillance System Hospitals (2002). Secular trend of hospital-acquired candidemia among intensive care unit patients in the United States during 19891999. Clin Infect Dis 35, 627-630.

Wey, S. B., Mori, M., Pfaller, M. A., Woolson, R. F. \& Wenzel, R. P. (1988). Hospital acquired candidaemia. The attributable mortality and excess length of stay. Arch Intern Med 148, 2642-2645. 